\title{
La nouvelle donne de la gouvernance régionale : les enjeux pour les femmes
}

\author{
Marielle Tremblay et Claude Gilbert \\ Groupe de recherche et d'intervention régionales, UQAC
}

Le concept de démocratie ne prendra un sens réel et dynamique que lorsque les orientations politiques et les législations nationales seront définies en commun par les hommes et les femmes, en prenant équitablement en compte les intérêts et le génie spécifique des deux moitiés de la population. Conseil interparlementaire, avril $1992^{1}$

\section{Introduction}

La gouvernance et le développement d'une communauté impliquent une pluralité d'acteurs. En intégrant des représentants du milieu communautaire et des groupes de femmes aux instances de gouvernance dans les années 1980 et 1990, l'État québécois avait démontré une volonté d'inclusion des forces vives d'une société aux débats sur son devenir. Cependant, les modalités de gouvernance prévues dans la loi 34 (Loi sur le ministère du Développement économique et régional et de la Recherche) accentuent le rôle des élus des divers paliers de gouvernement au détriment des autres acteurs de la société civile. Ce faisant, cette loi modifie considérablement le portrait de la gouvernance dans les régions.

En effet, la loi 34 instaure les Conférences régionales des élus (CRÉ), consacrant du même coup le rôle prépondérant des élus et élues municipaux en ce qui a trait au développement régional. Ces CRÉs assumeront les mêmes mandats que les Conseils régionaux de développement (CRD). Une distinction importante doit être apportée, cependant. Alors que les CRD étaient principalement constitués de personnes repré- sentants les divers milieux sur un même territoire, les CRÉs sont essentiellement composées de personnes élues au niveau politique municipal. Ce changement est de nature à renforcer la démocratie de type représentatif. Or, cela va à l'encontre d'une tendance observée au Québec depuis une vingtaine d'années par laquelle la gouvernance privilégiait une démocratie de type social. Dans ce dernier modèle, les principaux acteurs sociaux d'un milieu donné sont représentés, dans une perspective d'équité et de justice. On pourrait dire que le type de démocratie ayant donné naissance aux CRD relevait de la démocratie sociale en raison de la très large participation des divers acteurs de la société civile au débat politique sur le développement d'une région.

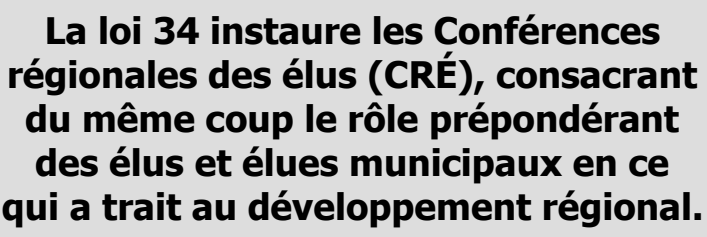
vernance à partir d'une composante fondamentale de la société civile de la région du Saguenay-Lac-SaintJean, c'est-à-dire les femmes. Les données ont été tirées de recherches menées dans la région au cours des dernières années ${ }^{2}$.

\section{Femmes, gouvernance et démocratie}

Les dispositions de la loi 34 soulèvent des questions sur la place des femmes dans la gouvernance, le développement des régions et la prise en compte de leurs intérêts spécifiques aux diverses instances régionalisées.

Du point de vue des femmes, la dynamique du développement n'est pas neutre: elle est sexuée, tout 
comme les conséquences sociales qui en découlent. Les intérêts des femmes, comme groupe social, n'y sont et n'y seront pris en compte que si elles réussissent à imposer leurs propres priorités. Une conception holistique du développement intègre l'importance du social et du politique et rend compte de la complexité de l'expérience des femmes. Cette approche situe le mouvement des femmes comme un acteur et un agent de définition des priorités d'utilisation des ressources collectives. Elle considère que les groupes de femmes produisent un discours et des pratiques de développement axés sur les besoins et les intérêts stratégiques des femmes comme groupe social. Ainsi, selon cette approche, non seulement les femmes doivent-elles produire une réflexion critique sur l'impact qu'ont sur leurs conditions de vie et de travail les décisions de développement, mais elles doivent travailler à en redéfinir les enjeux en fonction de leurs propres intérêts. Leur non-intégration dans les priorités et les projets de développement a, sur leurs conditions de vie, une série d'impacts négatifs, allant de la détérioration de leur condition économique jusqu'au renforcement des rapports inégalitaires hommes/femmes.

Le rôle des femmes dans la gouvernance repose sur plusieurs bases, dont le développement social. Ce concept fait référence à :

[...] la mise en place et [au] renforcement, au sein des communautés, dans les régions et à l'échelle de la collectivité, des conditions requises pour permettre, d'une part, à chaque individu de développer pleinement ses potentiels, de pouvoir participer activement à la vie sociale et de pouvoir tirer sa juste part de l'enrichissement collectif, et, d'autre part, à la collectivité de progresser socialement, culturellement et économiquement, dans un contexte où le développement économique s'oriente vers un développement durable, soucieux de justice sociale. ${ }^{3}$

La communauté et la société civile sont les acteurs obligés d'une telle conception du développement social. En effet, l'amélioration des conditions d'existence et de la qualité de vie pour les communautés et les individus passe par des politiques permettant, par exemple, la préservation de l'environnement et la réorientation des actions et des interventions. L'État joue ici un rôle de premier plan, bien sûr. Mais les citoyens sont aussi considérés comme des producteurs de développement en tant qu'interlocuteurs de l'État et du marché au sein de rapports politiques. Ces citoyens, regroupés en des associations et organismes divers portant leurs intérêts et besoins contribuent ainsi au développement de leur communauté non pas sur une base individuelle, comme le suggère le libéralisme, mais en tant que participants à une démocratie permettant la concertation des acteurs collectifs d'une société.

\section{Le rôle des femmes dans la gouvernance repose sur plusieurs bases, dont le développement social.}

Le développement social concerne ainsi l'amélioration de toutes les facettes et dimensions des conditions de vie des individus et des milieux. Il implique des relations microsociales harmonieuses et des relations macrosociales équitables, particulièrement en ce qui a trait à la richesse, à la démocratie, à la liberté, à l'accès aux services, etc. On comprendra alors que le développement social interpelle immédiatement le type de démocratie exercée dans la société. La démocratie représentative, où l'exercice de concertation est assumé soit par les représentants élus, soit par les parties concernées, présente des lacunes relativement à la mobilisation d'une communauté. On lui reprochera, bien sûr, d'être en butte à une crise de crédibilité des institutions politiques traditionnelles. Mais pour les fins de notre démonstration, nous mettrons plutôt l'accent sur un problème à caractère structurel, soit la difficulté des instances de démocratie représentative à prendre en compte l'ensemble des acteurs constituant l'organisation même d'une société : les divers milieux socio-économiques, les personnes plus fragiles, les personnes exclues, les femmes, etc. Afin de pallier cette lacune, l'État québécois a favorisé le développement de structures faisant appel à des principes de démocratie sociale. C'est ainsi que des associations ou groupes ont obtenu des sièges votants dans certaines instances dont les décisions avaient des conséquences immédiates en termes de concertation de la communauté, comme les Conseils régionaux de développement (CRD).

La démocratie sociale repose sur une certaine conception de la citoyenneté. Ce concept est généralement associé à l'usage des droits civiques en vue d'une participation aux différents scrutins. Mais de plus en 
plus, la citoyenneté quitte le champ réducteur de la démocratie représentative pour celui plus large de la démocratie sociale. Dans ce contexte, elle inclut la participation à des instances associatives ou institutionnelles (ex. comités de quartier, manifestations publiques de protestation, etc.) ${ }^{4}$. On dira alors qu'il s'agit d'une citoyenneté active, car elle concerne les contributions à la collectivité comme l'engagement civique, l'entraide et la participation. La citoyenneté active reconnaît donc la participation reconnue des femmes au développement et à la gouvernance d'une communauté.

\section{Le Saguenay-Lac-Saint-Jean a le plus fort pourcentage d'épouses sans revenu d'emploi $(37,2 \%)$ de toutes les régions du Québec (26,5\%).}

La contribution des femmes à la gouvernance et au développement des communautés repose donc sur des bases solides au plan théorique. Dans la même foulée, la réflexion récente en sciences politiques et études féministes a permis d'identifier deux stratégies permettant aux femmes d'intervenir directement dans les modèles de développement au plan régional ${ }^{5}$. La première consiste en une " politique de la présence ». En vertu de cette stratégie, les femmes réclament une représentation paritaire dans les instances de gouvernance. Cela implique aussi que des sièges leur soient spécialement attribués dans les structures de consultation et de décision. Par exemple, dans certaines instances régionales de gouvernance, cette attribution a pris la forme d'un "siège-femme ", ou "siège condition féminine » sur les conseils d'administration des CRD. Par contre, en ce qui a trait à la représentation paritaire des femmes dans les diverses instances de gouvernance, les mécanismes actuels ne le permettent guère, si ce n'est la présence plus soutenue de femmes à certaines commissions, conseils ou comités. L'autre stratégie est celle de la «politique des intérêts ». Celle-ci préconise que les intérêts des femmes, manifestés par les groupes de femmes d'un milieu donné, soient pris en compte dans les politiques de développement de nos communautés. Cette stratégie suppose une démocratisation des objets de l'intervention publique pour que celle-ci prenne en compte les femmes et que leurs intérêts, préoccupations, besoins, analyses et enjeux soient inscrits dans les politiques qui nous concernent toutes et tous en tant que collectivité. Son but est la promotion de la reconnaissance des intérêts spécifiques des femmes et la redéfinition, en fonction de ces intérêts, des moyens et des fins de l'utilisation des ressources collectives ou, en d'autres mots, du développement.

On comprendra la nécessité de la présence des femmes dans les instances de gouvernance, et de leur présence comme porteuses des intérêts des femmes, par une brève analyse des conditions socio-économiques des femmes de la région du Saguenay-LacSaint-Jean ${ }^{6}$. En effet, sous plusieurs aspects, les femmes de la région accusent des retards comparativement aux hommes de la même région ou aux femmes de l'ensemble du Québec. Il en est ainsi de la participation au marché du travail, qui est moindre pour les femmes de la région (47\%) que les hommes de la région $(68,2 \%)$ et que les femmes de l'ensemble du Québec $(54,6 \%)$. Les femmes de la région ont un plus haut taux de travail à temps partiel $(37,4 \%)$ que les hommes de la région $(14,1 \%)$ et que les femmes de l'ensemble du Québec (30,6 \%). Le Saguenay-LacSaint-Jean a le plus fort pourcentage d'épouses sans revenu d'emploi $(37,2 \%)$ de toutes les régions du Québec (26,5\%). Le revenu d'emploi des femmes de la région est moindre (16 258 \$) que celui de la moyenne féminine québécoise (19 484 \$) et que celui des hommes de la région (29 $328 \$$ ). La région compte aussi plus de ménages pauvres dirigés par une femme $(41 \%)$ que la moyenne québécoise $(39 \%)$. La scolarité des femmes de la région est aussi moindre que celle de l'ensemble des femmes du Québec, car la proportion de femmes ayant neuf ans et moins de scolarité est plus élevée au Saguenay-Lac-Saint-Jean $(18,2 \%)$ qu'ailleurs au Québec (16,3\%). La région compte une plus grande proportion de femmes de 65 ans et plus vivant sous le seuil de faible revenu que d'hommes. Cette dernière situation est accentuée dans la zone Chicoutimi-Jonquière.

De plus, on constate que les femmes sont peu présentes dans les instances de gouvernance. Ainsi, $27 \%$ des membres des commissions du CRCD étaient des femmes en 2001. Elles pouvaient être parfois majoritaires sur certaines commissions, mais souvent, elles étaient quasi absentes de plusieurs autres. Cette situation se reproduit dans la plupart des autres lieux décisionnels, qu'il s'agisse des cadres supérieurs de la fonction publique $(18,5 \%)$, des mairies $(11 \%)$, des conseils municipaux $(21,5 \%)$ et des postes de préfets de Municipalités régionales de comtés (MRC) $(0 \%)$ 
et des postes au sein de tous les Centres locaux de développement (CLD) de la région $(24,1 \%)$. Cela soulève des questions quant à la capacité d'une instance de gouvernance à pouvoir intervenir de façon équitable sur le développement d'une communauté.

Afin de promouvoir un changement de donne relativement à la place des femmes dans le développement régional et local, l'État québécois a ajouté, en 1997, une cinquième orientation à la politique en matière de condition féminine qui porte précisément sur la place des femmes dans le développement des régions ${ }^{7}$. De façon plus explicite, cette orientation énonçait la volonté d'une représentation équitable des femmes au sein des instances locales et régionales, d'une part, et la nécessité d'une prise en compte des intérêts et des réalités des femmes par les instances locales et régionales, d'autre part. Cette mesure s'inscrit dans un paradigme de démocratie sociale parce qu'elle permet d'introduire les intérêts des femmes dans des instances telles que les CRD et ce, malgré la sous-représentation des femmes dans certains lieux traditionnels de gouvernance comme les conseils municipaux. Cependant, les changements des dernières années ont consacré un retour vers ces formes traditionnelles de gouvernance, générant ainsi un recul démocratique.

Un cas récent permettra d'illustrer ce constat, celui du processus décisionnel de financement des entreprises d'économie sociale au Saguenay-Lac-Saint-Jean.

À la suite du Sommet socio-économique de 1996, le gouvernement québécois réaménageait une partie de ses programmes de façon à favoriser l'émergence d'entreprises d'économie sociale partout au Québec. Dès 1997, des Comités régionaux d'économie sociale avaient le mandat de promouvoir et de financer des entreprises dans les régions. Pour les gestionnaires des programmes gouvernementaux présents dans les comités régionaux, l'économie sociale constituait une continuité de la mission de leur ministère. Par exemple, le ministère de la Sécurité du revenu évaluait les projets soumis en fonction de leur potentiel à permettre l'intégration au travail de bénéficiaires de l'aide sociale. Pour sa part, le Secrétariat au développement des régions mettait l'accent sur la création d'emplois viables dans des régions lourdement affectées par le chômage. Ce phénomène s'expliquait par le fait qu'une bonne part des budgets disponibles pour le financement de projets d'économie sociale provenait d'instances gouvernementales et qu'à ce titre, il s'ac-

compagnait souvent des normes imposées par ces instances pour la distribution des fonds.

\section{Lors de l'exercice d'évaluation des projets et d'attribution du financement de 1997, les comités régionaux d'économie sociale ont joué un rôle déterminant.}

Présentes dans les comités régionaux d'économie sociale, les représentantes des groupes de femmes portaient une vision de l'économie sociale fortement marquée par les revendications de la Marche des femmes contre la pauvreté de 1995 et reprises dans le rapport Entre l'espoir et le doute (1996) du Comité d'orientation et de concertation sur l'économie sociale. Dans leur évaluation des projets devant faire l'objet d'un financement d'implantation de la part des comités régionaux d'économie sociale, elles ont fait jouer plusieurs critères. Les projets soumis devaient permettre de répondre à des besoins sociaux, principalement par la production de services accessibles pour tous les membres d'une communauté. Les services offerts devaient favoriser l'autonomie des membres de la communauté plutôt qu'une dépendance à l'égard de ces mêmes services. Les organismes soutenus devaient être autonomes face aux intérêts du secteur privé et au contrôle de l'État. Les représentantes des groupes de femmes cherchaient aussi à favoriser, dans le cadre de chaque projet, le développement de processus de décision démocratiques où personnel employé, promoteur-es et usager-ères seraient impliqué-es. Les projets devaient aussi être conçus dans la perspective de donner la primauté aux personnes dans la gestion des surplus et revenus. Les emplois créés devaient être durables, de qualité, avec un salaire décent et offerts aux femmes. Ces critères ont fait l'objet de nombreuses transactions lors des travaux des comités régionaux d'économie sociale, tous les membres ne partageant pas la même conception de l'économie sociale que les représentantes des groupes de femmes.

Lors de l'exercice d'évaluation des projets et d'attribution du financement de 1997, les comités régionaux d'économie sociale ont joué un rôle déterminant. Ils ont eu la latitude de se doter de critères régionaux en ce qui a trait au salaire de base, à la durée du financement ainsi qu'au caractère marchand des projets. La présence de représentantes de groupes de femmes a 
généré une dynamique permettant d'ouvrir le travail de ces comités aux revendications des femmes. Lors de leur travail d'évaluation des projets, les comités ont été sensibilisés au potentiel de développement d'infrastructures sociales d'emplois de qualité pour les femmes. Les participantes ont reconnu l'existence d'un espace de négociation sur les comités, bien que cette sensibilisation ait été l'objet d'un travail constant de la part des représentantes de groupes de femmes. Cette présence significative a pu prendre forme, car les représentantes des groupes de femmes disposaient d'un poids suffisant pour faire valoir leurs préoccupations. Ainsi, lors de la première année d'exercice du comité régional d'économie sociale du SaguenayLac-Saint-Jean en 1997, il y avait quatre sièges occupés par les représentantes des groupes de femmes sur les dix sièges constituant le comité. Cette présence s'explique sans doute par le momentum donné par la Marche des femmes contre la pauvreté ainsi que par la visibilité des femmes dans les travaux du Comité d'orientation et de concertation et du Groupe de travail sur l'économie sociale de 1996. La présence des représentantes des groupes de femmes sur ces comités régionaux d'économie sociale tombait à point, car ils avaient alors un rôle décisionnel dans l'analyse des projets et l'attribution des budgets d'implantation. Malgré les nombreuses transactions ayant eu cours lors des travaux des comités, qui ont parfois obligé les représentantes des groupes de femmes à s'accommoder des critères de fonctionnement de leurs divers visà-vis, il est possible de dire que la présence de ces dernières a eu un impact indéniable dans la configuration de l'économie sociale dans une région comme le Saguenay-Lac-Saint-Jean.

\section{La diminution progressive du rôle des groupes de femmes dans la gouvernance régionale, observée à la fin des années 1990 et confirmée par la loi 34, constitue un recul démocratique pour les femmes.}

Trois ans plus tard, en 2000, ce portrait avait considérablement changé. Les comités régionaux d'économie sociale se sont élargis, leur composition s'est renouvelée et leur rôle a été changé. En effet, dans le contexte de la réforme du ministre Chevrette, ces comités régionaux d'économie sociale ont eu pour fonction d'aviser les CRD en ce qui a trait à l'économie sociale dans le développement d'une région. Au SaguenayLac-Saint-Jean, la composition du comité régional d'économie sociale est passée de dix sièges à vingtquatre. Dans le renouvellement de ces sièges, ceux occupés par les groupes de femmes sont passés de quatre à deux. Le rapport de force a donc été considérablement changé. Ces changements ont eu comme conséquence d'amoindrir la capacité des femmes à promouvoir leur vision du développement social dans la région. Une partie des fonds désignés au soutien d'initiatives en économie sociale a été affectée au Fonds de lutte à la pauvreté. Les Centres locaux de développement (CLD), répartis selon les territoires des Municipalités régionales de comtés (MRC), ont obtenu la gestion des budgets destinés à soutenir le développement d'entreprises d'économie sociale. Les conseils régionaux de développement (CRD) jouaient dorénavant un rôle important dans la planification du développement régional. Or, malgré leur importance relative au sein de la Commission sectorielle d'économie sociale, les représentantes des groupes de femmes ont été minoritaires dans la plupart des instances de gouvernance, du moins au Saguenay-Lac-Saint-Jean. De plus, les femmes devaient désormais promouvoir leur conception de l'économie sociale face aux représentants de pouvoirs locaux pour qui, bien souvent, le développement de services pour les personnes de notre société en butte à des exclusions ne constitue pas une priorité.

La situation décrite ci-haut illustre bien certains enjeux relatifs à la démocratie. En effet, les élus locaux, légitimement désignés dans leur communauté, portent avec eux des intérêts particuliers, influencés par les modèles sociaux dominants. Ce modèle ne permet pas aux autres groupes sociaux de participer au débat public de la même façon. C'est pourquoi l'État québécois avait introduit un principe de démocratie sociale dans certaines instances de gouvernance. Les effets en ont été positifs, car cela a permis à divers acteurs de la société civile d'avoir voix au chapitre, dont les femmes. Peu présentes dans les instances traditionnelles de gouvernance (ex. les conseils municipaux), elles ont pu défendre leurs intérêts et gagner de la crédibilité en étant présentes sur celles créées dans les années 1980 et 1990 (ex. les CRD). La diminution progressive du rôle des groupes de femmes dans la gouvernance régionale, observée à la fin des années 1990 et confirmée par la loi 34, constitue un recul démocratique pour les femmes. 


\section{Conclusion}

Dans les pages qui précèdent, nous avons voulu démontrer que le retour vers une démocratie représentative instauré par la loi 34 semble présenter toutes les apparences d'un déficit démocratique pour nos communautés régionales. Le développement ne peut se faire sans la participation des femmes. Or, leur rôle dans les instances de gouvernance régionale est atténué et ce, malgré que l'État québécois ait déjà reconnu la contribution des acteurs de la société civile selon le principe de la citoyenneté ${ }^{8}$. Or, le repli vers une forme de gouvernance basée uniquement sur une démocratie de type représentatif a des conséquences importantes sur les femmes. Ces dernières sont en minorité parmi les édiles municipaux et préfets de MRC, sans compter la députation à l'Assemblée nationale. Elles sont donc en position très largement minoritaire au sein de la Conférence régionale des élus. De plus, la diminution du rôle des organismes communautaires dans les instances locales et régionales de gouvernance contribue à l'exclusion des femmes, car elles forment une part importante du personnel et des usagères de ces groupes. Pour les femmes, la loi 34 instaure une double exclusion de la gouvernance régionale.

Ce déficit de démocratie pose aussi la question du recul de l'État sur ses engagements en matière de développement. La cinquième orientation de la Politique en matière de condition féminine concernant le développement local et régional insistait sur la nécessité d'intégrer les femmes dans les lieux de gouvernance afin de contrer leur sous-représentation dans les conseils municipaux, centres et comités de tous genres. La loi 34 se démarque de cette orientation politique, car elle pose les conditions d'une exclusion des femmes des instances de gouvernance.

\section{Nous avons voulu démontrer que le retour vers une démocratie représentative instauré par la loi 34 semble présenter toutes les apparences d'un déficit démocratique pour nos communautés régionales.}

Cette situation fait en sorte que la loi 34 peut être critiquée à partir du concept de «bonne gouvernance » qui s'impose au plan mondial ${ }^{9}$. Ce concept est maintenant intégré aux programmes des Nations Unies en matière de développement et a trait aux conditions démocratiques devant être observées par les gouvernements. Selon les Nations Unies, la gouvernance doit être participative, durable, légitime et acceptée par la population, transparente, opérant sur la règle du droit, imputable, etc. Soulignons de façon particulière deux de ces conditions : la promotion de l'équité et de l'égalité ainsi que la promotion de l'équilibre entre femmes et hommes. On conviendra que les habitudes de gouvernance développées au Québec rencontrent certainement les standards onusiens. D'ailleurs, la cinquième orientation de la Politique en matière de condition féminine s'inscrivait dans cette optique de bonne gouvernance. Mais en affaiblissant la position des femmes au sein du débat public par la loi 34, l'État québécois se soustrait à deux conditions de bonne gouvernance. En effet, dans sa composition même, la Conférence régionale des élus n'annonce aucune mesure permettant d'assurer l'équilibre entre femmes et hommes. De plus, l'absence des groupes de femmes dans cette instance ne constitue pas un signal favorable pour la promotion de l'équité et de l'égalité. Cette exclusion des femmes, et du milieu communautaire, du débat public nous apparaît préjudiciable pour le développement de nos collectivités.

\section{Notes et références}

1 Conseil interparlementaire, Plan d'action : pour remédier aux déséquilibres actuels dans la participation des hommes et des femmes à la vie politique, Genève, Union interparlementaire, 1994.

2 AFÉAS, Denise Côté, Éric Gagnon, Claude Gilbert, Nancy Guberman, Francine Saillant, Nicole Thivierge et Marielle Tremblay (1998). Qui donnera les soins? Les incidences du virage ambulatoire et des mesures d'économie sociale sur les femmes du Québec / Who Will Be Responsible for Providing Care? The Impact of the Shift to Ambulatory Care and of Social Enonomy Policies on Quebec Women, Ottawa, Condition féminine Canada, mars, p. x- 134 p. / p. xiv-122.

Tremblay, Marielle et Claude Gilbert (2001). « Femmes et économie sociale au Saguenay-Lac-Saint-Jean », dans Anita Caron, Anick Druelle, Nadine Goudreault, Charlotte Thibeault et Marielle Tremblay (dir.), L'économie sociale et la lutte contre l'appauvrissement des femmes, Montréal, Relais-femmes, p. 49-58.

Tremblay, Marielle et Pierre-André Tremblay (1998). Les contributions sociales et économiques des organismes 
communautaires de la Corporation de développement communautaire $d u$ Roc, Chicoutimi, Groupe de recherche et d'intervention régionales, mars, $218 \mathrm{p}$.

3 Conseil de la santé et du bien-être, cité dans Deena White, Bruno Jean et Maurice Lévesque, Les conceptions du développement social, le point de vue des acteurs, p. 6.

Gautrat, Jacques (2001). «La qualité demain : une dimension citoyenne », dans J.-L. Laville et M. Nyssens (dir.), Les services sociaux entre associations, marché et État. L'aide aux personnes âgées, Paris, La Découverte / MAUSS / CRIDA, p. 186-191.

5 Philips, Anne (1991). Engendering Democracy, Cambridge, Polity Press; Id., « Dealing with difference : a politics of ideas, or a politics of presence?, dans Seyla Benhabib (dir.), Democracy and Difference. Contesting the Boundaries of the Political, Princeton, NJ, Princeton University Press, p. 156-191, cité dans D. Masson, «Femmes, régions et démocratie : la présence et les intérêts », dans N. Thivierge et M. Tremblay (dir.), Régionalisation et démocratie: les défis d'une citoyenneté active pour les femmes, Montréal, Réseau féministe de chercheures et d'intervenantes pour un renouvellement des théories et des pratiques économiques et politiques pour la redistribution des richesses, p. 55-61.

Conseil du statut de la femme (2002). Les conditions de vie des femmes et le développement régional et local au
Saguenay-Lac-Saint-Jean, Québec, Conseil du statut de la femme, coll. «Femmes et développement des régions », mars. Les données sont basées sur le recensement de 1996 de Statistique Canada.

7 Secrétariat à la condition féminine (1997). La place des femmes dans le développement des régions. $5^{e}$ orientation, Québec, Gouvernement du Québec, 67 p. Les quatre autres orientations sont: 1) l'autonomie économique des femmes; 2) le respect de l'intégrité physique et psychologique des femmes; 3) l'élimination de la violence faite aux femmes; 4) la reconnaissance et la valorisation de l'apport collectif des femmes.

8 Conseil québécois de la santé et du bien-être (2001). L'appropriation par les communautés de leur développement, Québec, Conseil québécois de la santé et du bienêtre, p. 11, cité dans Hurtubise, Yves (2001). Action communautaire, pouvoir des citoyens et des communautés, Baie-Comeau, Université rurale québécoise, septembre.

9 Women's Political Participation and Good Governance: $21^{s t}$ Century Challenges, UNDP, 2000, p. 19, cité dans D. Lafontaine, «Régionalisation de deuxième type et gouvernance : un agenda de recherche et d'action pour les femmes ", dans N. Thivierge et M. Tremblay (dir.), Régionalisation et démocratie: les défis d'une citoyenneté active pour les femmes, Montréal, Réseau féministe de chercheures et d'intervenantes pour un renouvellement des théories et des pratiques économiques et politiques pour la redistribution des richesses, p. 13-49. 


\title{
Publicité
}

\section{Programmes de $2^{\mathrm{e}}$ cycle du DSEA}

\author{
(4 carrés)
}

